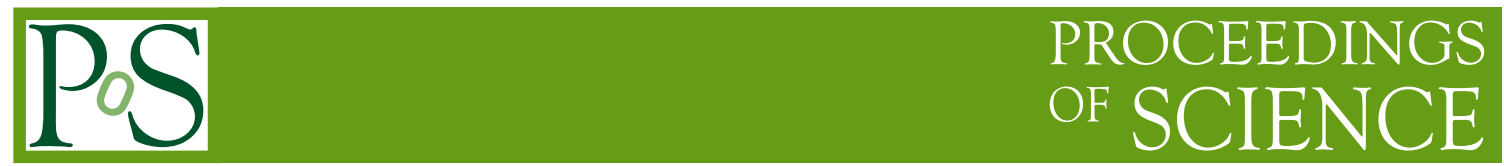

\title{
Null Tests of Time Reversal Invariance Using Neutrons
}

\section{William Snow* Indiana University}

E-mail: wsnoweindiana.edu

We discuss two methods using slow neutrons to search for interactions which violate both parity $(\mathrm{P})$ and time reversal $(\mathrm{T})$ symmetry. The first and most well-known example is the continuing effort to find an electric dipole moment for the neutron. Another possibility is to search for a parity-odd and time reversal-odd amplitude in polarized neutron optical transmission through polarized nuclei. Both of these observables are null tests for $\mathrm{P}$ and $\mathrm{T}$ violation. Both of these methods also have the potential to improve the sensitivity compared to current limits on $\mathrm{P}$ and $\mathrm{T}$ violating amplitudes in nucleon-nucleon interactions by about two orders of magnitude.

The 3rd International Symposium on "Quest for the Origin of Particles and the Universe" 5-7 January 2017

Nagoya University, Japan

*Speaker. 


\section{Introduction}

The search for new sources of $\mathrm{T}$ violation is one of the highest intellectual priorities in nuclear/particle/astrophysics. A. Sakharov [1] suggested that CP violation (and therefore time-reversal-invariance violation, assuming (PT invariance) is an essential requirement to produce the matter-antimatter asymmetry in the Big Bang. To date T violation has only been observed in $\mathrm{K}$ and $\mathrm{B}$ meson systems. However the strength of $\mathrm{T}$ violation in this system is several orders of magnitude too small to explain the cosmological data. New sources of $\mathrm{CP} / \mathrm{T}$ violation beyond the Standard Model are therefore suspected to exist to explain the observed matter-antimatter asymmetry of the universe. Many physicists have postulated theories which can produce more T violation through Beyond Standard Model (BSM) particles. Theoretical progress to identify the large number of possible sources for $T$ violation and to trace how such new sources would manifest themselves in low-energy observables has made it very clear that any single type of $T$ violation search cannot be equally sensitive to all possible mechanisms. It is therefore essential to pursue any experiments in different systems which can be realized with sufficient sensitivity to discover something new $[2,3])$.

The scientific justification to search for $\mathrm{T}$ violation in nucleons is very strong. Since the contribution to $\mathrm{T}$ violation in strongly-interacting systems of light quarks from the complex phase in the CKM matrix through loop effects is 5-6 orders of magnitude smaller than the present experimental upper bounds on T odd amplitudes, there is plenty of room to search for new sources of $\mathrm{T}$ violation in these systems. The present upper bound on the neutron EDM already strongly constrains theories beyond the Standard Model. Advances in lattice gauge theory now make it possible to actually calculate directly how a source of BSM T violation is manifested in a single nucleon or in a few-nucleon system [4, 5]. Present constraints on the neutron EDM already push the limits on the mass scale for new $\mathrm{T}$ violation physics above $10 \mathrm{TeV}$. Improvements in the sensitivity of null tests for $\mathrm{P}$ and $\mathrm{T}$ violation using neutrons by 1-2 orders of magnitude can tightly constrain possible $\mathrm{T}$ violation in the Higgs sector [6,7], conclusively test the minimal supersymmetric model for electroweak baryogenesis [8,9], and tightly constrain model-independent analyses of $\mathrm{P}$ and $\mathrm{T}$ violation in combination with ongoing EDM searches in atomic systems [10]. Neutron EDM bounds are already sensitive enough to address the question of whether the baryon asymmetry was produced during the electroweak symmetry-breaking era [11]. T violation in different particle species and in different atomic and nuclear systems possesses different sensitivity to possible contributions from quark EDMs and color-EDMs, purely gluonic CP-violating interactions, and CP-violation in the lepton sector [10].

In this paper we concentrate specifically on the neutron system and in particular on two observables which are null tests for $T$ violation. One is an electric dipole moment for a neutron. The other is a T-odd term in the elastic forward scattering amplitude of polarized neutrons on polarized targets. In both cases an observable can be constructed which realizes the motion-reversed condition directly corresponding to the well-known property of the reversal of initial and final states produced in a $T$ transformation. In the case of a neutron electric dipole moment defined as the expectation value of an electric 
dipole moment operator in the rest frame of a particle, the initial and final states of the system are the same and so the $T$ transformation does not lead to an independent physical process. In the case of the forward elastic neutron scattering amplitude, since the state of the (polarized) target does not change one can realize the time-reversed process simply by passing the neutron beam backwards through the target with all spins reversed. Since the optical theorem relates the imaginary part of the forward scattering amplitude to the cross section, the cross section differences for these forward and reversed processes are proportional to amplitude differences due to a T-odd term in the interaction.

$T$ violation can in principle exist with or without accompanying violation of parity. In this paper we focus on null tests of $\mathrm{T}$ which are both $\mathrm{P}$-odd and T-odd. Indirect constraints on P-even and T-odd interactions from analyses of radiative corrections to constraints on P-odd and T-odd interaction from electric dipole moment searches [12] are generally much more stringent than the direct experimental constraints. We therefore do not consider Peven and T-odd interactions further in this paper.

\subsection{Current status of nEDM experiments}

The neutron electric dipole moment is sought through delicate interference experiments based on Ramsey's separated oscillatory field method. [13] In this method a polarized ensemble in the spin state $|\uparrow\rangle_{z}$ is immersed in both an electric field and a magnetic field along $z$. If only the magnetic field is present the neutron spin precesses at the Larmour frequency. A transverse RF magnetic field is applied to this ensemble for a time long enough to tip the neutron spin direction by $\pi / 2$ about the $\mathrm{z}$ axis, thereby producing a coherent superposition of spin states for all of the neutrons in the ensemble of the form $\frac{1}{\sqrt{2}}\left(\left|\uparrow>_{z}+\right| \downarrow>_{z}\right)$. An electric field is then applied to this ensemble along the $z$-axis, and if the neutron possesses an electric dipole moment the energy shift between the two components of this coherent superposition will develop a relative phase which grows linearly with time. Such a relative phase shift is equivalent to an additional rotation of the neutron spin. This additional rotation is read out experimentally by applying a second $\pi / 2$ pulse which is phase coherent with the original pulse and measuring the projection of the neutron spin along $\mathrm{z}$. By applying these two $\pi / 2$ pulses with slightly different time separations, one can map out an interference fringe pattern between the two spin states $|\uparrow\rangle_{z}$ and $|\downarrow\rangle_{z}$. With an appropriate choices of the time separation which corresponds to the locations of maximum slope of the interference pattern, one can maximize the sensitivity of the interference to the energy shift from an EDM as the direction of the electric field is changed.

The current experimental limit on the neutron EDM (nEDM), $d_{n}<2.9 \times 10^{-26} e \cdot \mathrm{cm}$ ( $90 \%$ C.L.), was set by an experiment performed at ILL by a group led by the University of Sussex [14]. A recent updated analysis gives $d_{n}<3.0 \times 10^{-26} e \cdot \mathrm{cm}(90 \%$ C.L.) [15]. This experiment was based on Ramsey's separated oscillatory field method as applied to polarized ultracold neutrons (UCN) trapped in a material bottle. The UCNs were polarized using a magnetized iron foil, stored and allowed to freely precess in a precession chamber with electric and magnetic fields, and the final polarization state was analyzed using the same iron foil. The experiment featured a "comagnetometer" based on polar- 
ized ${ }^{199} \mathrm{Hg}$ atoms which suppresses magnetic field noise. The upper bound on the ${ }^{199} \mathrm{Hg}$ EDM is so small that any observed EDM signal in this experiment would come from the neutron. This experiment was ultimately limited by neutron counting statistics. ILL also hosts a nEDM experiment conducted by a PNPI collaboration. This experiment employs a double chamber measurement cell with opposed electric fields in the two chambers, uses simultaneous analysis of both spin states after the free precession period, and external Cs magnetometers rather than comagnetometers to control magnetic field noise. The PNPI collabortion has recently published a new result $\left|d_{n}\right|<5.5 \times 10^{-26} e \cdot \mathrm{cm}$ (90\% C.L.) [16]. There is a plan to operate the PNPI experiment at a higher UCN flux position at the ILL.

Another experiment has been operating for a few years now at PSI. The PSI collaboration recycled and upgraded the NEDM experimental apparatus used by the Sussex-ILL experiment and brought it to their new UCN source. They also use the Ramsey method. They have implemented several upgrades to the original apparatus and have improved the magnetometry, magnetic shielding, and many other aspects of the experiment. The collaboration is expected to publish an nEDM result soon with a sensitivity of approximately $(1-2) \times 10^{-26} e \cdot \mathrm{cm}(90 \%$ C.L.).

In addition to PSI, many facilities (ILL, TRIUMF, TUM, LANL, PNPI, FLNP, JPARC, Osaka, Mainz, PULSTAR,...) have launched efforts to construct brighter UCN sources to lower the statistical error on UCN experiments such as the neutron EDM. These efforts take advantage of the new UCN production methods based on solid deuterium $\left(\mathrm{SD}_{2}\right)$ converters and on superthermal UCN production in superfluid helium. PNPI, Los Alamos, Mainz, and PSI constructed UCN sources based on $\mathrm{SD}_{2}$. Collaborations at some of these faciliites are pursuing R\&D activities to perform a neutron EDM experiment with sensitivity in the $\delta d_{n} \sim 10^{-27} e \cdot \mathrm{cm}$ regime. Most of these proposed searches are elaborations on the same Ramsey approach using UCN with various types of improvements in magnetic field shielding and control, better comagnetometry, and higher polarized UCN intensity.

One exception is a large cryogenic nEDM experiment with a sensitivity goal of $\delta d_{n} \sim$ $3 \times 10^{-28} e \cdot \mathrm{cm}$ based on a novel approach proposed by Golub and Lamoreaux [17] to be mounted at the Spallation Neutron Source at ORNL. In this experiment, UCN will be produced directly inside the apparatus from a cold neutron beam via the superthermal process in superfluid helium. This experiment does not use Ramsey's separated oscillatory field method. Instead polarized ${ }^{3} \mathrm{He}$ atoms are dissolved in the superfluid ${ }^{4} \mathrm{He}$ and serve as a co-magnetometer and as the precession frequency analyzer for the neutrons using the spin dependent nuclear reaction $n+{ }^{3} \mathrm{He} \rightarrow p+t$, which generates scintillation light in LHe. This approach also aims to take advantage of the much larger electric field compared to a room temperature vacuum-based apparatus that one can apply inside the liquid helium and the longer UCN storage time from suppressed upscattering at low temperatures. This experiment has the capability to reach $\mathscr{O}\left(10^{-28}\right) e \cdot \mathrm{cm}$ sensitivity. The experiment is currently in the Critical Component Demonstration (CCD) stage, in which prototypes of various sub-components of the experiment are built and demonstrated. When realized, this experiment has the capability to improve the sensitiviy to a neutron EDM by two orders of magnitude beyond the current limit. This would correspond to a sensitivity of the ratio of the P-odd T-odd amplitude in the neutron generating the EDM to the P-odd 
T-even effects known to exist from the Standard Model weak interactions between quarks of about $10^{-5}$.

\section{2. $T$ violation in polarized neutron optics}

The basic idea of this experiment is to investigate $T$ violation in neutron interactions with heavy nuclei at a compound nuclear p-wave resonance by searching for a term in the neutron forward scattering amplitude of the form $\vec{\sigma}_{n} \cdot\left(\vec{k}_{n} \times \vec{I}\right)$, where $\vec{\sigma}_{n}$ is the spin of the neutron, $\vec{k}_{n}$ is the neutron momentum, and $\vec{I}$ is the spin of the nucleus. This observable is both parity odd and time reversal odd. It is also a null test for T violation which is in principle free from the effects of final state interactions $[18,19,20]$. The highly excited states in heavy nuclei involved in this type of search offer a qualitatively different environment from the nuclear ground states probed by electric dipole moment experiments. One can take advantage of the tremendous amplification of symmetry violation in certain compound nuclei such as ${ }^{139} \mathrm{La},{ }^{131} \mathrm{Xe}$, and ${ }^{81} \mathrm{Br}$ where parity violating asymmetries of $2-10 \%$ have already been measured, and of our theoretical understanding of the amplification mechanism, which also works for P-odd and T-odd amplitudes. The ratio of the P-odd and T-odd amplitude to the P-odd amplitude on the same $\mathrm{p}$-wave resonance is quite insensitive to unknown properties of the compound resonant states involved. The estimate of the statistical uncertainty that could be achieved in such an experiment after $10^{7} \mathrm{sec}-$ onds of data at a MW-class short pulse neutron spallation source implies that one can measure the ratio of the P-odd and T-odd amplitude to P-odd amplitude with $1 \cdot 10^{-5}$ sensitivity, which translates into an improved sensitivity to P-odd and T-odd neutron-nucleus interactions of about 2 orders of magnitude [21,22, 23]. This sensitivity is therefore comparable to that being proposed in the future for the most ambitious neutron EDM searches described above.

Compound nuclear resonances which would greatly amplify the size of P-odd and Todd interactions have already been identified in previous experiments. Amplifications of $P$-odd neutron amplitudes by factors of $10^{5}-10^{6}$ above the $10^{-7}$ effects expected for weak NN amplitudes compared to strong NN amplitudes have already been observed [24] in certain heavy nuclei such as ${ }^{139} \mathrm{La},{ }^{131} \mathrm{Xe}$, and ${ }^{81} \mathrm{Br}$ on $p$-wave resonances in the $\mathrm{eV}$ energy range. The mechanism for this amplification has been understood theoretically for decades: in fact the amplified effects were predicted theoretically before they were measured experimentally. The same resonance amplification factor will apply to a $P$-odd and $T$-odd amplitude up to factors of order unity associated with the states connected by the different operators. One can form a dimensionless ratio $\lambda_{P T}=\frac{A_{P T}}{A_{P}}$ of the T-odd, P-odd asymmetry $A_{P T}$ of interest to the measured P-odd asymmetry $A_{P}$ at the position of the enhanced p-wave resonance energy. Since this ratio involves expectation values in the same compound nuclear wave functions it possesses a clean theoretical interpretation.

The Neutron OPtics Time Reversal EXperiment (NOPTREX) collaboration which has formed to pursue this experiment argues that the time is now ripe to pursue this experiment because of three key developments: (1) New theoretical work has sharpened our understanding of the potential reach of an experiment of this sort and clarified the range 
of possible sources of $T$ violation, revealing a wide variety of possible P-odd T-odd interactions between nucleons. (2) A new approach to the measurement technique has recently been proposed. Although the idea for an experiment of this type is far from new, the great majority of proposed methods have exhibited, upon further analysis, excessive sensitivity to the relative alignment of the neutron and target polarizations in the final polarization analysis step which has been employed in almost all previous proposals. The two key aspects of the new proposal which differ from the great majority of previous approaches are (a) to involve only one neutron beam polarizer and a polarized target and forego any downstream analysis of the polarization, and (b) to explicitly realize the motion-reversed condition corresponding to the time reversal transformation through the mechanical rotation of the apparatus. (3) New technology makes a very sensitive experiment possible. Intense sources of pulsed epithermal neutrons are now available. The separation of neutron energies by time-of-flight from pulsed neutron sources allows a powerful search for systematic errors by looking on and off the neutron resonance energy at both the transmitted and scattered neutrons. The technologies for both the production of epithermal polarized neutrons using polarized ${ }^{3} \mathrm{He}$ neutron spin filters and for the production and control of polarized nuclei of the relevant species have both greatly improved. The explosive development of polarized neutron imaging promises to realize the ability to perform 3D imaging of internal magnetic fields and nuclear polarization with enough spatial resolution to diagnose possible sources of systematic error from polarized target nonuniformities.

The experiment will require a large flux of $e V$ polarized neutrons, a polarized nuclear target, and a high count rate time of flight neutron detector for detection of zero angle elastically scattered neutrons with sharp neutron energy resolution. The experiment will also require the ability to flip neutron polarization, nuclear target polarization, and neutron momentum direction simultaneously. NOPTREX proposes a rotating apparatus to flip the neutron momentum direction. To flip the neutron polarization, we will perform a spin flip of the ${ }^{3} \mathrm{He}$ polarization. The ${ }^{3} \mathrm{He}$ and nuclear target polarization can be flipped by applying a small resonant radio frequency transverse field, and simultaneously flip of the B field adiabatically.

Groups at KEK, Kyoto University [25], and PSI [26] achieved substantial ( 50\%) polarizations of ${ }^{139} \mathrm{La}$ nuclei in lanthanum aluminate crystals in volumes as large as $10 \mathrm{cc}$. The $0.734 \mathrm{eV}$ resonance in ${ }^{139} \mathrm{La}$ has a P-odd longitudinal asymmetry of $9.5 \%$ and is therefore a good candidate for the first experiment. Measurements at JPARC are investigating the nuclear spectroscopy on this resonance to assess the experimental sensitivity. If one uses ${ }^{139} \mathrm{La}$ nuclei in the form of lanthanum aluminate crystals, the aluminum nuclei will also be polarized. Using the NMR data from the experimentally achieved value of ${ }^{139} \mathrm{La}$ polarization of $47.5 \%$, one can show [27] that the nuclear pseudomagnetic field inside the crystal is reduced by the polarized aluminum nuclei.

\section{Summary}

Searches for new sources of $\mathrm{T}$ violation are important to pursue to address various 
unsolved issues in nuclear/particle/astrophysics. We described two efforts to search for new sources of time reversal violation using slow neutron measurements which are null tests for $\mathrm{T}$. The upper bound on the neutron electric dipole moment already constrains many BSM theories. Many groups propose to improve the sensitivity of the neutron EDM search, and the most ambitious project two orders of magnitude of improvement. We also described the improved prospects to search for a P-odd T-odd term in the neutron forward scattering amplitude in polarized neutron optics on a p-wave resonance in a polarized target, which is also a null test for T. Such an experiment has never been performed, but the statistical accuracy which could be achieved now is comparable in sensitivity to the most ambitious future nEDM projects. Recent advances in polarized neutron technology and in the analysis of systematic effects have encouraged the NOPTREX collaboration to pursue this measurement. Even if one or both of these experiments realize their sensitivity goals and see no effects, the tight constraints such null results would place on BSM T violation should provide an essential clue about the new physics which must exist.

\section{Acknowledgments}

The work of W. M. Snow was supported by US National Science Foundation grant PHY-1614545, by the Indiana University Center for Spacetime Symmetries, and by the Indiana University Collaborative Research and Creative Activity Fund of the Office of the Vice President for Research.

\section{References}

[1] A. D. Sakharov, Violation of CP Invariance, C Asymmetry, and Baryon Asymmetry of the Universe, Pisma Zh. Eksp. Teor. Fiz. 5, 32 (1967).

[2] M. Pospelov and A. Ritz, Electric dipole moments as probes of new physics, Ann. Phys. 318, 119 (2005).

[3] J. Engel, M. J. Ramsey-Musolf and U. van Kolck, Electric Dipole Moments of Nucleons, Nuclei, and Atoms: The Standard Model and Beyond, Prog. Part. Nucl. Phys. 71, 21 (2013).

[4] T. Bhattacharya, V. Cirigliano, R. Gupta, E. Mereghetti and B. Yoon, Dimension-5 CP-odd operators: QCD mixing and renormalization, Phys. Rev. D 92, 114026 (2015).

[5] T. Bhattacharya, V. Cirigliano, R. Gupta, H. W. Lin and B. Yoon, Neutron Electric Dipole Moment and Tensor Charges from Lattice QCD, Phys. Rev. Lett. 115, 212002 (2015).

[6] S. Inoue, M. J. Ramsey-Musolf and Y. Zhang, CP-violating phenomenology of flavor conserving two Higgs doublet models, Phys. Rev. D 89, 115023 (2014).

[7] Y. T. Chien, V. Cirigliano, W. Dekens, J. de Vries and E. Mereghetti, Direct and indirect constraints on CP-violating Higgs-quark and Higgs-gluon interactions, JHEP 1602, 011 (2016).

[8] V. Cirigliano, Y. Li, S. Profumo and M. J. Ramsey-Musolf, MSSM Baryogenesis and Electric Dipole Moments: An Update on the Phenomenology, JHEP 1001, 002 (2010).

[9] Y. Li, S. Profumo and M. Ramsey-Musolf, A Comprehensive Analysis of Electric Dipole Moment Constraints on CP-violating Phases in the MSSM, JHEP 1008, 062 (2010) 
[10] T. Chupp and M. Ramsey-Musolf, Electric Dipole Moments: A Global Analysis, Phys. Rev. C 91, 035502 (2015).

[11] D. E. Morrissey and M. J. Ramsey-Musolf, Electroweak baryogenesis, New J. Phys. 14, 125003 (2012).

[12] W. C. Haxton, A. Hoering, and M. J. Ramsey-Musolf, Constraints on T-odd and P-even hadronic interactions from nucleon, nuclear, and atomic electric dipole moments, Phys. Rev. D50, 3422 (1994).

[13] N. F. Ramsey, A Molecular Beam Resonance Method with Separated Oscillating Fields, Phys. Rev. 78, 695 (1950).

[14] C. A. Baker et al., Improved Experimental Limit on the Electric Dipole Moment of the Neutron, Phys. Rev. Lett. 97, 131801 (2006).

[15] J. M. Pendlebury et al., A Revised Experimental Upper Limit on the Electric Dipole Moment of the Neutron, Phys. Rev. D 92, 092003 (2015).

[16] A. P. Serebrov et al., New measurements of neutron electric dipole moment with double chamber EDM spectrometer, Phys. Rev. C 92, 055501 (2015).

[17] R. Golub and S. K. Lamoreaux, Neutron Electric Dipole Moment, Ultracold Neutrons, and Polarized ${ }^{3} \mathrm{He}$, Phys. Rep. 237, 1 (1994).

[18] V. P. Gudkov, On the test of CP violation models in neutron reactions, Phys. Lett. B243, 319 (1990).

[19] V. P. Gudkov and Y. H. Song, Discovery potential in a search for time-reversal invariance violation in nuclei, Hyperfine Interact. 214, 105 (2013).

[20] J. D. Bowman and V. P. Gudkov, Search for time reversal invariance violation in neutron transmission, Phys. Rev. C90, 065503 (2014).

[21] V. E. Bunakov and V. P. Gudkov, Parity violation and related effects in neutron induced reactions, Nucl. Phys. A401, 93 (1983).

[22] V. P. Gudkov, On CP violation in nuclear reactions, Phys. Rept. 212, 77 (1992).

[23] A. G. Beda and V. R. Skoy, Current status of research on T invariance in neutron-nuclear reactions, Phys. Part. Nucl. 38, 1063 (2007).

[24] G. E. Mitchell, J. D. Bowman, and H. A. Weidenmuller, Parity violation in the compound nucleus, Rev. Mod. Phys. 71, 445 (1999).

[25] Y. Takahashi, T. Yabuzaki, and H. M. Shimizu, Possible nuclear polarization of ${ }^{139} \mathrm{La}$ in $\mathrm{Nd}-3+$ LaAlO-3 for the test of time reversal invariance, Nucl. Instrum. Meth. A336, 583 (1993).

[26] P. Hautle and M. Iinuma, M. Dynamic nuclear polarization in crystals of $\mathrm{Nd}-3+\mathrm{LaAlO} 3, a$ polarized ${ }^{139}$ La target for a test of time-reversal invariance, Nucl. Instrum. Meth. A440, 638 (2000).

[27] V. P. Gudkov, Recoil effects and CP violation in neutron scattering, Phys. Rev. C46, 357 (1992).

[28] G. E. Mitchell, J. D. Bowman, S. I. Penttila, and E. I. Sharapov, Parity violation in compound nuclei: experimental methods and recent results, Phys. Rep. 354, 157 (2001). 